\title{
La importancia de la mujer en el desarrollo. Análisis de los Objetivos de Desarrollo Sostenible con perspectiva de género
}

\author{
The importance of women in development. Analysis of \\ Sustainable Development Goals with a gender perspective
}

\author{
DiANA M. Verdiales LóPEZ* \\ Universidad Rey Juan Carlos
}

ORCID ID: 0000-0003-3275-2058

Recibido: $16 / 06 / 2020$

Aceptado: 6/09/2020

doi: https://doi.org/10.20318/femeris.2020.5765

\begin{abstract}
Resumen. La participación de la mujer en los distintos sectores económicos, políticos, sociales y ambientales constituye la pieza clave para alcanzar el desarrollo humano sostenible que tanto anhelamos. Su fortaleza, inteligencia, capacidad para gestionar los recursos, así como su capacidad de cuidado de los demás y del medio que la rodea la convierten en el motor de desarrollo humano sostenible. Sin embargo, uno de los obstáculos a los que se tienen que enfrentar millones de mujeres en todo el mundo es el escaso reconocimiento de sus derechos en todos los ámbitos, lo que la hace susceptible a padecer situaciones de discriminación, violencia y exclusión social. Si bien en los últimos 40 años se ha producido un gran avance en la incorporación de la perspectiva de género en las agendas internacionales, todavía existen importantes retos en torno a su participación igualitaria y plena en la sociedad internacional. Por ello, este trabajo tiene como propósito analizar los sucesos históricos que ha posibilitado el reconocimiento de los derechos de la mujer después distintos enfoques o metodologías, así como determinar la posible incorporación de la perspectiva de género en los 17 Objetivos de Desarrollo Sostenible (ODS), destacando la importancia de la participación de la mujer en cada uno de dichos objetivos. Todo ello basado en una metodología de análisis cuantitativo de revisión de bibliográfica existente sobre dicha temática y cuantitativo sobre la revisión de los indicadores contenidos en el documento de ONU MUJERES "Hacer las promesas realidad: la igualdad de género en la agenda 2030 para el desarrollo sostenible", publicado en el 2018.

Palabras clave: Objetivos de Desarrollo Sostenible, mujer y desarrollo, derechos de la mujer, perspectiva y enfoque de género, indicadores de género.
\end{abstract}

Abstract. Women's participation in the various economic, political, social and environmental sectors is the key to achieving the sustainable human development that we so long for.

\footnotetext{
*Doctora en Derechos Humanos, Paz y Desarrollo Sostenible por la Universidad de Valencia, España. Personal Docente Investigador de la Universidad Rey Juan Carlos (URJC). Responsable de Proyectos de Investigación y Cooperación del Centro de Estudios de Iberoamérica, URJC. Este trabajo se ha realizado en el marco del Grupo de Investigación de Alto Rendimiento en Libertad, Seguridad y Ciudadanía en el Orden Internacional (INTERCIVITAS) de la Universidad Rey Juan Carlos, del cual soy miembro.

diana.verdiales@urjc.es
} 
Its strength, intelligence, ability to manage resources, as well as its capacity to care for others and the environment around it make it the engine of sustainable human development. However, one of the obstacles that millions of women around the world face is the poor recognition of their rights in all areas, making it susceptible to situations of discrimination, violence and social exclusion. While there has been a major advance in gender mainstreaming in international agendas over the past 40 years, there are still significant challenges around their equal and full participation in international society. Therefore, this work aims to analyze the historical events that have enabled the recognition of women's rights after different approaches or methodologies, as well as to determine the possible mainstreaming of the gender perspective in the 17 Sustainable Development Goals (SDGs), highlighting the importance of women's participation in each of these objectives. All this based on an existing quantitative analysis methodology of literature review on this subject and quantitative on the review of indicators contained in the UN WOMEN document "Making Promises Reality: Gender Equality on the 2030 Agenda for Sustainable Development", published in 2018.

Keywords: Sustainable Development Goals, women and development, women's rights, gender perspective and approach.

\section{Introducción}

Tras más de 20 años desde la aprobación de la Declaración y Plataforma de Acción de Beijing (Beijing, 1995) ${ }^{1}$, podemos decir que el balance en la implementación de estrategias internacionales para la promoción de la igualdad de género y la eliminación de todo tipo de desigualdades sigue siendo negativo. Si bien, tras estas dos décadas de trabajo coordinado entre los diferente organismos internacionales en materia de género, se han registrado importantes avances como el aumento en un $20 \%$ de la participación de la mujer en los parlamentos nacionales y en el mercado laboral en general, así como su acceso a servicios básicos como la educación y la salud; es necesario enfatizar que siguen existiendo retos importantes para lograr que la participación de las mujeres en las diferentes esferas sociales, económicas, policitas y culturales se dé de forma igualitaria, reconocida y valorada.

Hasta la fecha no existe país en el mundo que haya logrado una plena igualdad de género, sino que se siguen observando altos niveles de desigualdad entre hombres y mujeres en los diferentes ámbitos económicos, políticos y sociales. Siendo necesario y urgente continuar trabajando en el acceso de las mujeres a un trabajo decente, a la eliminación de la brecha salarial por motivos de género, a lograr un equilibrio de la carga por trabajo doméstico o cuidados no remunerados, a eliminar cualquier tipo de violencia hacia la mujer, a trabajar en la educación sexual y reproductiva como parte del derecho a la salud, y a que la participación de la mujer en el ejercicio del poder y la toma de decisiones se incremente de forma más igualitaria en todo el mundo.

Es por todos sabido que la participación activa de la mujer en las diversas esferas de la sociedad es fundamental para el logro de un desarrollo sostenible. Se reconoce su liderazgo innato para promover acciones no solo en su entorno familiar sino también en su comunidad ${ }^{2}$. Por lo que a mediados de los años sesenta y setenta, cuando se empieza a

\footnotetext{
${ }^{1}$ Disponible en: https://beijing20.unwomen.org/ /media/headquarters/attachments/sections/csw/bpa_s_final_ web.pdf

${ }^{2}$ SCHOLTUS Silvia C. y DOMATO 0. (2015) "El rol protagónico de la mujer en el desarrollo sustentable de la comunidad". Apuntes Universitarios, p. 12
} 
analizar el factor humano en las teorías economista del desarrollo, se identifica a la mujer como pieza clave para el desarrollo y la desigualdad de género como el principal obstácu$l^{3}$. Sin embargo, pese a que reconocer la importante labor de la mujer en el desarrollo y la importancia de trabajar en la igualdad entre hombres y mujeres, el papel de la mujer ha quedado relegado, en un segundo plano, tras considerar su participación en función de la familia y la infancia ${ }^{4}$. Siendo éste uno de los grandes problemas de los planes y políticas de desarrollo implementadas alrededor del mundo, al considerar a la mujer como cuidadora de su núcleo familiar o como madre pobladora (en términos de reproducción) dejando de lado su identidad integral como mujer 5 .

Por ello, y bajo el lema "el empoderamiento de la mujer empodera a la humanidad" la Plataforma de Acción de Beijing pretende continuar promoviendo diversas acciones hasta lograr la igualdad de género, el empoderamiento de las mujeres y el reconocimiento de los derechos de todas la mujeres y niñas en el mundo, ya que continúa siendo la principal hoja de ruta para orientar la lucha contra los obstáculos y restricciones que impiden el empoderamiento de las mujeres en todo el mundo. Por ello, trabaja arduamente en la promoción de las doce esferas prioritarias que, desde hace más de 20 años y hasta la actualidad, ha estado apoyando para alcanzar los objetivos y metas que en cada una de ellas se han establecido. Dichas esferas abarcan: la pobreza, la educación y la capacitación, la salud, la violencia contra la mujer, los conflictos armados, la economía, el ejercicio del poder y la toma de decisiones, los mecanismos instituciones para el adelanto de la mujer, los derechos humanos, los medios de difusión, el medio ambiente y la niña. Basados en los objetivos y metas establecidas en cada una de las 12 esferas prioritarias establecidas en la Declaración y Plataforma de Beijing, los gobiernos en coordinación con la sociedad civil, han implementado acciones concretas para cambiar la realidad de millones de mujeres alrededor del mundo ${ }^{6}$.

Aunado a estas acciones, desde la Organización de las Naciones Unidas se aprobó el compromiso internacional de continuar trabajando por una sociedad más justa y equitativa bajo el lema "sin que nadie se quede atrás"7. Por lo que, desde los Objetivos de Desarrollo Sostenible (ODS, 2015-2030) plasmados en la Agenda 2030 se da por formalizado dicho compromiso internacional para dar continuidad a las acciones emprendidas en los Objetivos de Desarrollo del Milenio (ODM, 2000-2015). Tanto en los ODM como en los ODS la perspectiva de género ha estado presente. En los ODM se abordó esta temática en el Objetivo 3 para trabajar por la igualdad entre géneros y la autonomía de la mujer, teniendo como meta principal la eliminación de las desigualdades entre los sexos en la enseñanza primaria y secundaria, monitoreando la proporción de mujeres entre los emplea-

\footnotetext{
${ }^{3}$ HEIKEL, Maria Victorial (1994) “Mujer, población y desarrollo”, Población y Desarrollo, №. 7, p. 36

${ }^{4}$ BERNAL MARTÍNEZ DE SORIA, Aurora y CARRICA OCHOA, Sarah (2014) "Mujer, desarrollo y Educación para el Desarrollo", Revista Edetania: estudios y propuestas socio-educativas, p. 186.

${ }^{5}$ RODRÍGUEZ MARTÍNEZ, Rocio (1994) "Mujer y desarrollo: reflexiones sobre el feminismo del Norte y el desarrollo del Sur: estudio especial sobre la mujer en América Latina” América latina hoy: Revista de ciencias sociales, Vol. 9, p. 31.

${ }^{6}$ NACIONES UNIDAS (1994) "Declaración y Plataforma de Acción de Beijing. Declaración política y documentos resultados de Beijing+5”, Nueva York, U.S.A.

7 ASAMBLEA GENERAL DE NACIONES UNIDAS (2015) "Transformar nuestro mundo: la Agenda 2030 para el Desarrollo Sostenible”, Resolución 70/1, 25 de septiembre de 2015, p. 13.
} 
dos remunerados en el sector no agrícola, así como la proporción de puestos ocupados por mujeres en los parlamentos nacionales.

En los ODS la perspectiva de género está contenida más ampliamente en el Objetivo 5 , para lograr la igualdad entre los géneros y empoderar a las mujeres y las niñas, aunque 9 Objetivos más abordan las cuestiones de género de manera transversal. En lo que respecta al ODS 5 se formularon 6 metas y tres recomendaciones relativas a la eliminación de todas las formas de discriminación contra todas las mujeres y las niñas en todo el mundo; la eliminación de todas las formas de violencia contra todas las mujeres y las niñas; la eliminar de todas las prácticas nocivas, como el matrimonio infantil, precoz y forzado y la mutilación genital femenina; el reconocimiento del trabajo doméstico no remunerado; la participación plena y efectiva de las mujeres y la igualdad de oportunidades de liderazgo a todos los niveles decisorios; y acceso universal a la salud sexual y reproductiva y los derechos reproductivos ${ }^{8}$.

El presente trabajo aborda, en consecuencia, las principales problemáticas que siguen obstaculizando el desarrollo integral de las mujeres y su participación igualitaria en las diferentes esferas de la sociedad. Haciendo un recorrido por los ODS y la incorporación en éstos del enfoque transversal de género, y enfatizando en la importancia de la participación mujer en todos los ámbitos para la generación de un desarrollo integral y sostenible que haga de la sociedad internacional una sociedad más justa, humana y equitativa.

\section{Aproximación a los derechos de las mujeres en el sistema internacional}

La necesidad de introducir la perspectiva de género en los diferentes ámbitos económicos, políticos, sociales, culturales viene apoyada por la creciente desigualdad que aqueja a la sociedad en la mayoría de los países del mundo. Por ello es importante señalar que, aunque los primeros estudios sobre género se originaron a mediados del siglo XIX, los avances en la materia no han logrado mejorar las condiciones de vida de la mayoría de las mujeres en el mundo. En concreto, el concepto de género como sabemos, es un concepto relacionado con las relaciones socioeconómicas y culturales entre personas de diferente sexo $^{9}$. Es decir, refleja la manera en la que nos relacionados con función de nuestro sexo y lo que socialmente se espera de nosotros, haciendo referencia a los roles ${ }^{10}$, estereotipos y responsabilidades asignadas por la sociedad tanto social como culturalmente por el hecho de ser hombre o mujer ${ }^{11}$.

\footnotetext{
${ }^{8}$ ONU MUJERES (2018) “Hacer las promesas realidad: la igualdad de género en la agenda 2030 para el desarrollo sostenible”, Estados Unidos de América, p. 86.

9 CALATRAVA, J. (2002), "Mujer y Desarrollo Rural en la globalización: de los proyectos asistenciales a la planificación de género", Revista Globalización y Mundo Rural, No. 803. p. 73. Cfr. en: https://www.who.int/topics/ gender/es/\#: :text=G\%C3\%A9nero.\%20El\%20g\%C3\%A9nero\%20se\%20refiere $\% 20 \mathrm{a} \% 20$ los\%20 conceptos,los $\% 20$ hombres\%20y\%20las\%20mujeres $\% 20$ que $\% 20$ favorecen $\% 20$

${ }^{10}$ Existe una amplia descripción de los roles de género, los cuales son considerados como un conjunto de valores, actitudes y conductas establecidas para cada género por la sociedad, siendo éstas: rol reproductivo, rol productivo y rol comunitario. Mayor información ver en: AECID, (2015), "Guía de la AECID para la Transversalización del Enfoque de Género", p. 19.

${ }^{11}$ Cfr. AECID, (2015), “Guía de la AECID para la Transversalización del Enfoque de Género”, p. 14.
} 
En ese sentido se han llevado a cabo diversas estrategias y enfoques para incorporar las cuestiones de género en los distintos ámbitos, entre los que podemos destacar los siguientes ${ }^{12}$ :

- El Enfoque del Bienestar. Desarrollado durante los años 50, este enfoque pone el énfasis en la mejora de las necesidades de las mujeres generadas desde su posición como madres y esposas y las coloca en el centro de los proyectos de desarrollo como beneficiarias pasivas.

- Enfoque de las Necesidades Básicas: Corriente surgido en los años 70 de enfoque para el desarrollo basado en las necesidades humanas en contraposición a las corrientes de crecimiento económico ${ }^{13}$. En este enfoque la mujer es definida como sujeto de desarrollo y vista como proveedora de bienes y servicios ${ }^{14}$.

- El Enfoque Mujeres en el Desarrollo (MED): Este enfoque desarrollado durante los años 70 fue promovido principalmente por las organizaciones de mujeres y para el desarrollo las cuales pusieron de manifestó los resultados desiguales entre los hombres y las mujeres. El MED representa a las mujeres como miembros productivos de la sociedad, promoviendo el acceso de las mujeres a los recursos productivos para promover la igualdad. Sin embargo, este enfoque no toma en consideración las relaciones de género, de poder, de socialización y los roles entre hombres y mujeres para la generación de desarrollo ${ }^{15}$.

- El Enfoque Género en el Desarrollo (GED): Desarrollado durante los años 80, este enfoque analiza e intenta transformar aquellas relaciones de poder desiguales que obstaculizan el disfrute equitativo y pleno de las mujeres en todos los ámbitos del desarrollo.

- Enfoque de Género (mainstreaming) $\underline{16}$ : Enfoque desarrollado a finales de los años 90 y principios de 2000, el cual pretende identificar y tener en cuenta las necesidades y principales problemáticas que afectan tanto a hombres como mujeres. La incorporación transversal de dicho enfoque (mainstreaming) pretende integrar la perspectiva de género tanto en el ámbito político como técnico, así como realizar los cambios funcionales y estructurales necesarios para mejorar el desarrollo y evaluación de los procesos de toma de decisiones.

- El Enfoque basado en los Derechos Humanos (EBDH): Desarrollado por la Oficina del Alto Comisionado de Naciones Unidas para los Derechos Humanos tras el segundo taller inter-agencial realizado en Stanford en 2003, donde más de 15

\footnotetext{
${ }^{12}$ Ibíd., p.16.

${ }^{13} \mathrm{Cfr}$. http://www.dicc.hegoa.ehu.es/listar/mostrar/154\#: :text=El\%20enfoque\%20de\%20las\%20necesidades\%20 b\%C3\%A1sicas\%20no\%20ha,la\%20situaci\%C3\%B3n\%20actual\%20de\%20satisfacci\%C3\%B3n\%20de $\% 20$ las $\% 20$ necesidades.

${ }^{14}$ HEIKEL, Maria Victorial (1994) “Mujer, población y desarrollo”, Población y Desarrollo, №. 7, p. 36-37.

${ }^{15}$ FERNÁNDEZ SAAVEDRA Ana G. y DEMA MORENO Sandra (2018) "La integración de la perspectiva de género en la gestión del riesgo de desastres: de los ODM a los ODS” Revista Internacional de Cooperación y Desarrollo Vol. 5 No. 1, pág: 33

${ }^{16}$ Fundación Mujeres (2003), “Guía para la elaboración de proyectos desde una perspectiva de género", Fondo Social Europeo y Gobierno del Principado de Asturias, p. 43.
} 
agencias de Naciones Unidas pusieron de manifesto su interés para incluir los derechos humanos en las políticas públicas de todos los países del mundo, promoviendo la elaboración de un entendimiento común sobre un enfoque basado en los Derechos Humanos. Dicho enfoque se ha constituido como un marco conceptual para el desarrollo humano basado en las normas internacionales de derechos humanos, desde el punto de vista normativo y operativo para la promoción y protección de estos derechos al poner en el centro de los problemas de desarrollo las desigualdades, las practicas discriminarlos y el reparto injusto del poder, los cuales obstaculizan el progreso del desarrollo humano. Es preciso señalar, no obstante, que tanto el EBDH como el Enfoque de Género son complementarios y que se recomienda la incorporación transversal del enfoque de género en el enfoque de Derechos humanos para dar un mayor énfasis al cumplimiento a las cuestiones relacionadas con la igualdad y el empoderamiento de las mujeres.

Al igual que los enfoques elaborados para promover la igualdad de género, las normativas internacionales en la materia también han ido evolucionando con el paso de tiempo. Una buena clasificación sobre la evolución de los derechos de las mujeres la podemos encontrar en la Guía de la AECID para la Transversalización del Enfoque de Género ${ }^{17}$ la cual señala como primera etapa de visibilización de las desigualdades el periodo comprendido entre 1945 y 1962, donde quedan incorporadas dichas desigualdades en la Carta de las Naciones Unidas (1945), que señala expresamente, por primera vez, la discriminación por motivo de sexo constituyéndose como el primer instrumento jurídico en afirmar la igualdad de todos los seres humanos; en la creación de la Comisión Jurídica y Social de la Mujer (1946) cuyo propósito principal era la elaboración de informes y recomendaciones sobre las problemáticas más urgentes en relación a los derechos de la mujer, dando promoción de dichos derechos en las áreas policía, económica, social, educativa, etc.; y, con alcance regional europeo, en el Tratado de Roma (1957) que apunta una de las normativas internacionales más relevantes en materia de protección de la mujer y la igualdad de género.

En una segunda etapa, se habla sobre el Germen de la Institucionalidad en materia de los derechos de la mujer, comprendido entre 1963 y 1975, donde aparecen como principales acciones en materia de género la Declaración para la Eliminación de la Discriminación contra la Mujer (1967) la cual sienta las bases para la formulación de políticas, cambio de actitudes, compromisos políticos e instituciones para la igualdad de género; y la organización de la Primera Conferencia Mundial del Año Internacional de la Mujer en México (1975) la cual adoptó un plan de acción plurianual para la implementación de estrategias transnacionales en materia de igualdad de género ${ }^{18}$.

Durante el periodo comprendido entre 1976-1985 se produjo un cambio sobre el rol de la mujer a nivel internacional, por lo que durante este periodo se llevaron a cabo dos grandes acciones: la aprobación en 1979 de la Convención sobre la Eliminación de to-

\footnotetext{
${ }^{17}$ AECID, (2015), “Guía de la AECID para la Transversalización del Enfoque de Género", p. 25.

${ }^{18}$ BERNAL MARTÍNEZ DE SORIA, Aurora y CARRICA OCHOA, Sarah (2014) “Mujer, desarrollo y Educación para el Desarrollo", Revista Edetania: estudios y propuestas socio-educativas, p. 186.
} 
das las Formas de Discriminación contra la Mujer (CEDAW) la cual se constituye como la Carta Magna de los derechos de las mujeres al ser un instrumento jurídico vinculante enunciando los principios internacionalmente reconocidos sobre los derechos de las mujeres; y la celebración en 1980 en Copenhague de la Conferencia Mundial del Decenio de las Naciones Unidas para la Mujer la cual hizo visible la diferencia existente entre los derechos garantizados (igualdad formal) y la capacidad de las mujeres para ejercer dichos derechos (igualdad real), adoptándose para ello la Plataforma de Acción de Copenhague.

Durante 1985 y 1995, se lleva a cabo un reforzamiento por parte de los organismos de apoyo a las mujeres, así como la incorporación de dos conceptos nuevos: el empoderamiento y el mainstreaming (TG). Durante este periodo, tiene lugar en Nairobi 1985 la Conferencia Mundial para el Examen y la Evaluación de los logros del Decenio de las Naciones Unidas para la Mujer, en la cual se ponen de manifiesto las diferencias existentes en materia de igualdad exhortando a los gobiernos a promover acciones para asegurar la paz, el desarrollo y la igualdad bajo tres ejes principales: la igualdad en la participación social, en la participación política y en la toma de decisiones; la Conferencia Mundial de los Derechos Humanos celebrada en 1993 en Viena la cual considera como elementos centrales de la agenda de los derechos humanos, los derechos de las mujeres y apoyando la erradicación de la violencia de género; la celebración en el Cairo en 1994 de la Conferencia Internacional sobre Población y Desarrollo la cual incorpora la concepción del desarrollo basado en las personas incluyendo aspectos como las desigualdades de género, la reducción de la pobreza, entre otros; la Cumbre Mundial sobre Desarrollo Social en 1995 la cual reconoce a las mujeres como el pilar fundamental para la erradicación de la pobreza; y, en ese mismo año, la Cuarta Conferencia Mundial sobre la Mujer en Beijing la cual se considera como la agenda clave en materia de género al reunir a un número elevado e líderes políticos y representantes de ONG's de todo el mundo. En dicha Conferencia se adoptó la Plataforma de Acción de Beijing (PAdB) la cual pretende garantizar las condiciones de las mujeres en 12 esferas consideramos como críticas, las cuales han sido descritas anteriormente.

Posteriormente se desarrolla una nueva etapa con la adopción de la Agenda para los Objetivos de Desarrollo del Milenio (ODM) y las estrategias para la eficacia de la ayuda durante el periodo comprendido entre 1997 y el 2015. Las principales acciones llevadas a cabo durante este periodo fueron: la adopción, en el seno de la Unión Europea, del Tratado de Ámsterdam en 1997 en el cual se hace hincapié en la igualdad de género en el ámbito laboral; la Declaración de los Objetivos del Milenio en 2000 la cual comprendía el desarrollo de 8 ODM. En dichos objetivos se introdujo tímidamente el concepto de género, siendo el Objetivo 3 el que con más detalle abordaba esta problemática al promover la igualdad de género y el empoderamiento de la mujer, y en menor media en el Objetivo 5 el cual introdujo, a partir de 2005 , la cuestiones relativas a la salud sexual y reproductiva; la adopción de la Resolución 1325 del Consejo de Seguridad en ese mismo año en la cual se exhortaba a los gobiernos a incorporar el enfoque de género para el campo de las operaciones de mantenimiento de la paz; el Acuerdo ACP-CE en el 2000 que instaba a los gobiernos de países en desarrollo a incorporar el enfoque de género en sus políticas nacionales; la Carta de los Derechos Fundamentales de la Unión Europea en ese mismo año la cual tiene entre sus 
principios fundamentales la igualdad de género; el Reglamento relativo al Fomento de la Igualdad entre el Hombre y la Mujer en la Cooperación al Desarrollo en el 2004 donde se propone la integración horizontal del enfoque de género en las acciones emprendidas en la cooperación internacional al desarrollo; el Consenso Europeo para el Desarrollo en el 2006 que pone de manifiesto que la promoción de la igualdad de género y los derechos de la mujer son un derecho fundamental y una cuestión de justicia social; el Plan de Trabajo para la Igualdad entre las Mujeres y los Hombres (2006-2010) que destaca la necesidad de continuar trabajando para mejorar la promoción de la igualdad de género en los ámbitos políticos, desarrollando actividades y medidas específicas para ello; el Tratado de Lisboa (2007) que manifiesta la importancia de la inclusión de la igualdad de género en la políticas de la UE; las Agendas de la Eficacia de la Ayuda de Paris (2005), Accra (2008) y Busán (2011); y el Plan de Acción para la Igualdad de Género y el Empoderamiento de las Mujeres en el Desarrollo (2010-2015). Desde la Declaración de París de 2015, se puso de manifiesto la importancia de la inclusión de los derechos humanos, la igualdad de género y la sostenibilidad ambiental para potenciar un efecto duradero en las vidas de las mujeres, hombres y niños, sin embargo estos aspectos no fueron ampliamente desarrollados en esta declaración. Sin embargo, fue en la Declaración de Accra donde más de 200 organizaciones defensoras de los derechos de las mujeres promulgaron una declaración del Foro de Mujeres con propuestas concretas para la aplicación de estrategias de transversalización el enfoque de género; y en la Declaración de Busán se puso el énfasis en que tanto la igualdad de género como el empoderamiento de las mujeres es una cuestión fundamental para lograr resultados en materia de desarrollo; el Pacto Europeo por la Igualdad de Género (2011-2020) que muestra un especial interés en la transversalización del enfoque de género en las políticas gubernamentales; y la Declaración Política del vigésimo aniversario de la Declaración y Plataforma de Acción de Beijing (2015) que toma en consideración la incompleta labor desarrollada en los ODM y para hacer frente a las áreas no consideradas en la Declaración y Plataforma de Acción de Beijing hace un reconocimiento tras 20 años de su adopción, para la promoción de la aplicación plena y eficaz de la Declaración de Beijing, en particular, para su incorporación en los ODS sobre la igualdad de género y el empoderamiento de todas las mujeres y las niñas.

Finalmente, habría que señalar una última etapa post 2015 para la promoción de los derechos de la mujer y la igualdad de género, la cual queda comprendida, sobre todo, en las siguientes acciones: el II Plan de Acción de la Unión Europea para la Igualdad de Género y el Empoderamiento de las Mujeres en el Desarrollo (2015-2020) que destaca cuatro pilares fundamentales en materia de género: la integridad física y psicológica, los derechos económicos y sociales/empoderamiento, y la participación a la vez que enfatiza el desarrollo de un cuarto pilar relativo al cambio en la cultura institucional para la promoción de la igualdad; la Agenda 2030 para el Desarrollo Sostenible (20152030) en la cual las cuestiones relativas a la igualdad de género y el empoderamiento de las mujeres y las niñas queda comprendido específicamente en el Objetivo 5 y de forma transversal en los 16 Objetivos restantes. Dicho Agenda y sus objetivos serán desarrollados más ampliamente en el siguiente apartado. 


\section{Los objetivos de desarrollo sostenible con perspectiva de género: retos y estrategias}

Como se señala en la Agenda 2030 "el desarrollo solo será sostenible si los beneficios favorecen por igual a mujeres y hombres"19. Bajo esta premisa resulta evidente que sin la participación activa de la mujer en las diversas esferas de la sociedad no será posible alcanzar ese desarrollo humano sostenible que todos deseamos. Sobre todo, si tomamos en consideración el papel fundamental de la mujer en la educación y formación integral en cada uno de los miembros de su núcleo familiar, gestionando no solo los recursos económicos y materiales sino contribuyendo, de forma individualizada, a la formación de una serie de valores claves para la construcción de sociedades más justa, equitativas y solidarias, clave para un desarrollo humano sostenible. Es por ello que resultan de gran importancia la implementación y el alcance de los Objetivos de Desarrollo Sostenible, ya que no sólo contribuye a generar una visión global sobre las estrategias requeridas alcanzar un desarrollo humano sostenible, y específicamente aquellas relativas a las cuestiones de género, sino que permite trabajar a cada país, en particular y conjuntamente, hacia el logro de los 17 objetivos globales bajo las esferas sociales, económicas y políticas, mejorando con ello las condiciones de sus ciudadanos y, por ende, de la sociedad internacional.

Uno de los principales problemas identificados en el desarrollo e implementación de los ODM fueron los problemas relacionados con la escasez de datos suficientes y fiables para medir cada una de las acciones llevadas a cabo por la falta de indicadores específicos. En ese sentido, cuando se empezó a trabajar en la Agenda 2030, se tomaron en consideración todas aquellas áreas de mejora identificados tras finalizar el periodo de implementación de los ODM. Es por ello que se para los ODS se elaboró un marco de indicadores mundiales para facilitar la incorporación de las metas y acciones contenidas en cada uno de los 17 ODS en las agendas políticas y programas concretos de los países que se han adherido a esta iniciativa global. A pesar de que se han producido numerosas mejoras en los últimos 40 años en la generación de indicadores y estadísticas en cuestiones de género, la disponibilidad de los datos para dar seguimiento a las cuestiones de género, según las bases de datos mundiales, es apenas del $26 \%{ }^{20}$.

De los 232 indicadores mundiales establecidos para los 17 ODS, solo 54 de estos están relacionados con las cuestiones de género. De este número de indicadores relativos a las cuestiones de género podemos ver cómo están mayormente contenidos en 6 (ODS1, ODS3, ODS4, ODS5, ODS8 y ODS16) de los 17 ODS; en menor medida en 5 de éstos (ODS2, ODS10, ODS11, ODS13 y ODS17) y finalmente en 6 de los 17 ODS no se registra encuentra ningún indicador relativo a las cuestiones de género (ODS6, ODS7, ODS9, ODS12, ODS14 y ODS15). Sin embargo, de estos 54 indicadores solo se tiene información suficiente y periódica de 10 indicadores. Por lo que continúan existiendo restricciones para dar seguimiento a los

\footnotetext{
${ }^{19}$ ONU Mujeres, (2018) "Hacer las promesas realidad. La igualdad de género en la Agenda 2030 para el Desarrollo Sostenible", p. 1.

${ }^{20}$ ONU MUJERES (2018) "Hacer las promesas realidad: la igualdad de género en la agenda 2030 para el desarrollo sostenible", p. 48.
} 
avances de los ODS relativos a las cuestiones de género, siendo principalmente la cobertura desigual de los indicadores específicos de género, las lagunas en los datos de género (desigualdades cruzadas que sufren algunas mujeres y niñas) así como la baja calidad y comparabilidad de los datos disponibles entre los diversos países y periodos establecidos ${ }^{21}$.

Para evitar este tipo de restricción en los datos y de acceso a la información se requiere que se integre una perspectiva de género en los programas nacionales de estadística de cada país y se priorice en la recopilación de datos en tiempo y forma.

En la siguiente tabla podemos observar cómo se introduce la perspectiva de género en cada uno de los 17 ODS así como los indicadores específicos existentes en cada uno de ellos.

Tabla 1.

Fuente: elaboración propia con información obtenida de ONU MUJERES (2018) "Hacer las promesas realidad: la igualdad de género en la agenda 2030 para el desarrollo sostenible".

\begin{tabular}{|c|c|}
\hline ODS & PERSPECTIVA DE GÉNERO \\
\hline $1 \mathrm{FN}$ & $\begin{array}{l}1 \text { de cada } 5 \text { niñas en el mundo vive en hogares que sobreviven con menos de } 1,90 \text { dls diarios. } \\
\text { En todo el mundo hay } 122 \text { mujeres, de } 25 \text { a } 34 \text { años de edad, por cada } 100 \text { hombres que } \\
\text { viven en la pobreza. En América Latina esta proporción se eleva a } 132 \text { mujeres por cada } 100 \\
\text { hombres. La pobreza de las mujeres proviene de la desigualdad en el acceso a los recursos } \\
\text { económicos, a la segmentación en el mercado laboral, la brecha salarial, a la incapacidad } \\
\text { para heredar tierras o pedir préstamos y sobre todo por la falta de oportunidades para } \\
\text { continuar los estudios y la desigualdad de acceso a la protección social. } \\
\text { Indicadores específicos de género: } 1.1 .1,1.2 .1,1.2 .2,1.3 .1,1.4 .2 \text { y 1.b.1 }\end{array}$ \\
\hline & $\begin{array}{l}\text { Se estima que } 789 \text { millones de personas, el } 11 \% \text { de la población mundial, están desnutridas. } \\
\text { Las mujeres tienen más probabilidades que los hombres de padecer inseguridad alimenta- } \\
\text { ria en más de dos terceras partes de los países del mundo. Una tercera parte de todas las } \\
\text { mujeres con empleo en el mundo trabajan en la agricultura, pero sólo el } 15 \% \text { son propieta- } \\
\text { rias de tierras, lo que limita el acceso a la alimentación que producen. En época de crisis, la } \\
\text { discriminación por motivos de género hace que las mujeres y las niñas sean las primeras en } \\
\text { comer menos, a pesar de trabajar más para asegurar la alimentación. } \\
\text { Indicador específico de género: } 2.3 .2\end{array}$ \\
\hline & $\begin{array}{l}\text { Como es sabido las mujeres y los hombres, aunque tienen el mismo derecho a una vida } \\
\text { sana, tiene necesidades sanitarias diferentes. El embarazo y el parto suponen riesgos altos } \\
\text { para las mujeres y sus hijos. En todo el mundo mueren cada día } 840 \text { mujeres por causeas } \\
\text { relacionadas con el embarazo y el parto, alcanzando una cifra superior a las } 300.000 \text { muer- } \\
\text { tes de mujeres en } 2015 \text { por estos motivos. Son múltiples los factores que agravan las condi- } \\
\text { ciones de salud de la mujer, siendo algunos de éstos el elevado número de horas de trabajo } \\
\text { doméstico, la inseguridad de los entornos laborales y la violencia de género. } \\
\text { Indicadores específicos de género: } 3.1 .1,3.1 .2,3.3 .1,3.7 .1,3.7 .2 \text { y } 3.8 .1\end{array}$ \\
\hline $4 \mathrm{EDE}$ & $\begin{array}{l}\text { La educación proporciona además de conocimientos, oportunidades para mejorar las con- } \\
\text { diciones de vida, evitando en muchos casos el matrimonio infantil. La participación de las } \\
\text { niñas y mujeres en la educación sigue siendo desigual a la de los hombres. En educación pri- } \\
\text { maria la participación es similar, pero al entrar a la secundaria se incrementa considerable- } \\
\text { mente el abandono por parte de las niñas por motivos como el embarazo precoz, el trabajo } \\
\text { doméstico o el matrimonio infantil. Existen regiones en el mundo que cuentan con un } 48 \text { \% } \\
\text { de niñas sin escolarizar. Según el último informe de ONU Mujeres, } 15 \text { millones de niñas en el } \\
\text { mundo nunca tendrán la oportunidad ir a la escuela, frente a } 10 \text { millones de niños. } \\
\text { Indicadores específicos de género: } 4.1 .1,4.2 .1,4.2 .2,4.3 .1,4.5 .1,4.6 .1,4.7 .1 \text { y 4.a.1 }\end{array}$ \\
\hline
\end{tabular}

${ }^{21}$ Ibíd. 


\begin{tabular}{|c|c|}
\hline 5 IEUALADD & $\begin{array}{l}\text { Resulta imposible pensar en una igualdad efectiva entre hombres y mujeres si tomamos } \\
\text { en consideración que las mujeres continúan dedicando } 2.6 \text { veces más de tiempo que los } \\
\text { hombres a las tareas domésticas (suponiendo menos ingresos económicos y tiempo para } \\
\text { trabajos distintos o remunerados). Pese a que la violencia de género está considerada como } \\
\text { una de las violaciones de los derechos humanos más generalizada en el mundo, en la actua- } \\
\text { lidad } 1 \text { de cada } 5 \text { mujeres de } 87 \text { países del mundo ha sufrido algún tipo de violencia en todo } \\
\text { el mundo y } 49 \text { países continúan sin implementar leyes de protección contra la violencia de } \\
\text { género. Por otro lado, existen } 39 \text { países en el mundo que prohíben la igualdad de derechos } \\
\text { sucesorios entre hijos e hijas. Y } 15 \text { millones de niñas menores de } 18 \text { años en todo el mundo } \\
\text { no pueden disfrutar de su infancia porque son obligadas a contraer matrimonio. A pesar de } \\
\text { la aplicación de cuotas especiales sólo el } 23.1 \% \text { de las mujeres ocupan puestos parlamenta- } \\
\text { rios y menos de un tercio de puestos de dirección medio y alto. } \\
\text { Indicadores específicos de género: } 5.1 .1,5.2 .1,5.2 .2,5.3 .1,5.3 .2,5.4 .1,5.5 .1,5.5 .2,5.4 .1 \text {, } \\
5.5 .1,5.5 .2,5.6 .1,5.6 .2,5 . a .1,5 . a .2,5 . b .1 \text { y 5.c.1 }\end{array}$ \\
\hline 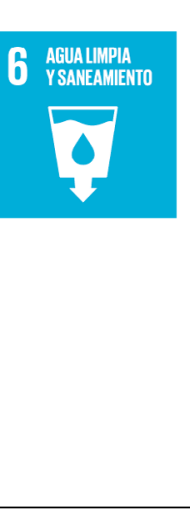 & $\begin{array}{l}\text { A pesar de que el agua es un bien imprescindible para la vida, en } 2015 \text { había } 2,100 \text { millones } \\
\text { de personas alrededor del mundo que carecían de ella. En el } 80 \% \text { de los hogares sin agua en } \\
\text { todo el mundo, son las mujeres y las niñas las responsables de la recogida del agua, siendo } \\
\text { un factor clave en el ausentismo y la deserción escolar. Como bien es sabido, la falta de agua } \\
\text { tiene consecuencias graves para la salud, por lo que resulta necesario continuar trabajando } \\
\text { para suministrar agua potable a las personas que carecían de este servicio básico. La fun- } \\
\text { ción de las mujeres en este objetivo es fundamental, ya que son ellas las que la mayoría de } \\
\text { las veces desempeñan las funciones de abastecimiento y preservación del agua, así como } \\
\text { del uso eficiente del mismo para el saneamiento. Asimismo, son ellas las que asumen el cui- } \\
\text { dado de los miembros de su familia y afrentan las amenazas que la falta de este bien puede } \\
\text { ocasionar en la salud. } \\
\text { Indicadores específicos de género: N/A }\end{array}$ \\
\hline & $\begin{array}{l}\text { En todo el mundo existen todavía 1,100 millones de personas que parecen de acceso a la } \\
\text { electricidad, por lo que tienen que hacer uso de combustibles sólidos tanto para cocinar } \\
\text { como para calentar sus hogares. En el 2012, murieron prematuramente cerca } 4,3 \text { millones } \\
\text { de personas por la contaminación del aire al interior de las viviendas, afectando mayorita- } \\
\text { riamente a mujeres y niñas ( } 6 \text { de cada 10). La recogida de dichos combustibles se realiza } \\
\text { mayoritariamente por mujeres, requiriendo para ello } 18 \text { horas semanales, lo que imposibi- } \\
\text { lita la participación plena de las niñas en las actividades escolares. Algunos indicadores re- } \\
\text { velan que las mujeres utilizan un } 22 \% \text { menos de energía lo que no solo permite un consumo } \\
\text { responsable, sino que son ellas las que producen los cambios de comportamiento diarios en } \\
\text { el interior de la familia para el uso responsable de los recursos energéticos. } \\
\text { Indicadores específicos de género: N/A }\end{array}$ \\
\hline $8 \underset{\mathrm{V}}{\mathrm{IR}}$ & $\begin{array}{l}\text { La participación de las mujeres en el mercado laboral sigue siendo desigual, ya que según } \\
\text { los datos la tasa laboral de las mujeres es del } 63 \% \text { frente al } 94 \% \text { de los hombres. Un factor } \\
\text { sigue siendo la segmentación profesional, que implica la dominación en ciertos puestos por } \\
\text { hombres. Asimismo, existe en la actualidad, una brecha salarial entre hombres y mujeres a } \\
\text { nivel mundial del } 23 \% \text {. Para alcanzar la equidad salarial se estima que deberán transcurrir } \\
70 \text { años más, continuando con las estrategias implementadas hasta ahora, salvo que se dé } \\
\text { un fuerte impulso para que dicha equidad sea posible en un menor plazo. } \\
\text { Indicadores específicos de género: 8.3.1, 8.5.1, 8.5.2, 8.7.1, 8.8.1, 8.8.2 y 8.9.2 }\end{array}$ \\
\hline 9 snow & $\begin{array}{l}\text { Un factor clave para el desarrollo sostenible es la implementación y desarrollo de industria, } \\
\text { infraestructura basada en la innovación y la investigación bajo métodos sostenibles. En este } \\
\text { sentido, la participación de la mujer en la industria, investigación o innovación sigue siendo } \\
\text { baja, ya que tan sólo un } 29 \% \text { de las mujeres de todo el mundo (una tercera parte de los } \\
\text { puestos mundiales) desarrolla labores de innovación e investigación. Sólo en } 1 \text { de cada } 5 \\
\text { países alrededor del mundo se ha logrado una representación equitativa en el ámbito de la } \\
\text { investigación. En cuanto a los sectores de la construcción, la fabricación y la energía existen } \\
\text { muy pocas mujeres encargadas para la toma de decisiones. } \\
\text { Indicadores específicos de género: N/A }\end{array}$ \\
\hline
\end{tabular}




\begin{tabular}{|c|c|}
\hline 10 REDUGGÓONDELAS & $\begin{array}{l}\text { La desigualdad provoca que las personas pobres carezcan de menos recursos y oportuni- } \\
\text { dades, generando diversas formas de exclusión y discriminación. Las políticas sociales y } \\
\text { económicas deben orientarse en reducir dichas desigualdades y favorecer la igualdad de } \\
\text { género. El problema de la desigualdad afecta tanto a los países en desarrollo como a los } \\
\text { desarrollados, ya que en éstos últimos la desigualdad aumentó un } 11 \% \text { entre } 1990 \text { y el } \\
\text { 2010. Dicha desigualdad se ve más agudizada en entre las mujeres y las niñas, implicando } \\
\text { no sólo una desigualdad no solo económica sino también de acceso a la salud, la educación } \\
\text { y el bienestar. Para reducir las desigualdades en el mundo, la Agenda } 2030 \text { promueve la } \\
\text { implementación de leyes, políticas y programas públicos basados en el enfoque de derechos } \\
\text { humanos, lo que implica la participación activa de la población, sobre todo de los grupos } \\
\text { más vulnerables. } \\
\text { Indicadores específicos de género: } 10.2 .1\end{array}$ \\
\hline & $\begin{array}{l}\text { Si tomamos en consideración que en el } 2030 \text { el } 60 \% \text { de la población mundial vivirá en las } \\
\text { ciudades, debemos pensar en los servicios básicos que éstas deberán disponer para poder } \\
\text { proporcionar condiciones de vida dignas y sostenibles para sus habitantes. Es por ello que } \\
\text { se deben crear espacios urbanos más seguros y ampliar los servicios básicos a todas zonas } \\
\text { habitables. Las condiciones deplorables e inseguras en las que se encuentran millones de } \\
\text { mujeres en las ciudades a nivel mundial dificultan su participación plena en los distintos } \\
\text { sectores de la sociedad. Pudiendo ser víctimas tanto por la inseguridad como por la discri- } \\
\text { minación laboral o el acceso a los servicios básicos (más de la mitad de las mujeres y niñas } \\
\text { que habitan en zonas urbanas carecen de agua potable o espacios adecuados de viviendas. } \\
\text { Indicadores específicos de género: } 11.2 .1,11.7 .1,11.7,2\end{array}$ \\
\hline & $\begin{array}{l}\text { A pesar del consumo excesivo y poco sostenible concentrado mayoritariamente en los } \\
\text { países desarrollados, existen } 767 \text { millones de personas en el mundo con dificultades para } \\
\text { adquirir los productos básicos y necesarios para vivir. Uno de los productos de mayor con- } \\
\text { sumo en todo el mundo es el vehículo privado, el cual es utilizado en mayor medida por los } \\
\text { hombres. Según los indicadores las mujeres dependen más del transporte público, lo que } \\
\text { contribuye no sólo a un consumo responsable sino al cuidado del medio ambiente. Desde } \\
\text { los ODS se promueve la modificación de los procesos de producción más sostenibles y la } \\
\text { reducción del consumo excesivo de los recursos naturales. } \\
\text { Indicadores específicos de género: N/A }\end{array}$ \\
\hline & $\begin{array}{l}\text { Como es bien sabido, los efectos ocasionados por el cambio climático, tales como aumento } \\
\text { de temperaturas, tormentas, sequias, etc. constituyen un riesgo cada vez mayor para todas } \\
\text { las personas. En este sentido y según el informe de ONU Mujeres las mujeres y las niñas tie- } \\
\text { nen un } 14 \% \text { más de probabilidad de morir durante una catástrofe natural que los hombres. } \\
\text { Las mujeres pueden ofrecer aportaciones muy valiosas para gestionar mejor los recursos } \\
\text { naturales y mitigar los riesgos ambientales, debido a su gran su experiencia y conocimiento } \\
\text { adquirido como tradicionales administradoras de los recursos naturales. Sin embargo, sólo } \\
\text { una de cada } 4 \text { mujeres en el mundo se dedica a la agricultura. Siendo muy baja su participa- } \\
\text { ción en la toma de decisiones sobre el cuidado del medio ambiente. } \\
\text { Indicadores específicos de género: } 13 . b .1\end{array}$ \\
\hline & $\begin{array}{l}\text { Como sabemos los océanos representan un } 70 \% \text { del planeta, sin embargo, la contaminación, } \\
\text { la acidificación o la diezma de las poblaciones de peces constituyen una grave problemática } \\
\text { para el mantenimiento y conservación de la vida marítima. Alrededor de } 1000 \text { millones } \\
\text { de personas dependen de los océanos, mares y recursos marítimos para sobrevivir (12\% } \\
\text { población mundial). El sector marítimo está constituido casi en su totalidad por hombres, } \\
\text { mientras que las mujeres desempeñan puestos secundarios y mal pagados, sin protección } \\
\text { laboral, de salud al estar la mayoría de ellas sin contratos. Sólo } 100 \text { empresas eran dirigidas } \\
\text { por mujeres en } 2016 \text {. Por lo que la participación de la mujer en el sector marítimo sigue } \\
\text { siendo desigual y son éstas las que enfrentan un mayor riesgo no sólo por las condiciones } \\
\text { laborales sino por la degradación de los océanos. } \\
\text { Indicadores específicos de género: Ninguno }\end{array}$ \\
\hline
\end{tabular}




\begin{tabular}{|c|c|}
\hline 15 VIDA & $\begin{array}{l}\text { Son más de } 1.600 \text { millones de personas las que dependen de los bosques y pese a ello, } \\
\text { entre el } 2010 \text { y el } 2016 \text { se perdieron 3,300 millones de hectáreas. Ello implica que muchas } \\
\text { personas, sobre todo mujeres, dediquen más horas al día recorriendo largas distancia para } \\
\text { abastecerse de combustibles, follaje o alimentos. Pese a los conocimientos de las mujeres } \\
\text { sobre los cuidados tradicionales de la tierra, a menudo son excluidas de la toma de decisio- } \\
\text { nes sobre el cuidado de los ecosistemas terrestres. Siendo las más afectadas por la falta de } \\
\text { recursos naturales por la pérdida de superficie forestal. } \\
\text { Indicadores específicos de género: Ninguno/ No dispone }\end{array}$ \\
\hline & $\begin{array}{l}\text { Las mujeres suelen estar escasamente representadas en la gobernanza de las institucio- } \\
\text { nes, lo que recrudece las disparidades de género. Para reducir y eliminar las injusticias, la } \\
\text { inestabilidad y la discriminación, las mujeres necesitan poder acudir a instituciones justas } \\
\text { y eficaces que proporcionan un acceso justo y equitativo a la justicia. Recordemos que sólo } \\
\text { un } 24 \% \text { de las mujeres ocupan escaños parlamentarios a nivel mundial. } \\
\text { Indicadores específicos de género: } 16.1 .1,16.1 .2,16.2 .2,16.2 .3,16.7 .1 \text { y } 16.7 .2\end{array}$ \\
\hline 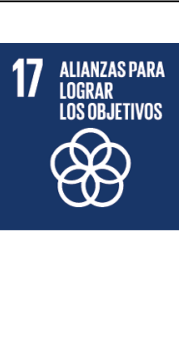 & $\begin{array}{l}\text { Se requieren de aspectos como la financiación, el comercio justo y equitativo, la tecnolo- } \\
\text { gía, las capacidades, las alianzas y la información, entre otros, para lograr una igualdad de } \\
\text { género y un desarrollo humano más sostenible. Por ello, la asistencia o ayuda al desarrollo } \\
\text { constituye actualmente una fuente de financiación que puede contribuir al logro de los ODS } \\
\text { en los países en desarrollo. Sin embargo, las cifras destinadas para este sentido son insufi- } \\
\text { cientes si se desea trabajar concienzudamente en la inclusión de la perspectiva de género } \\
\text { en los proyectos al desarrollo. } \\
\text { Indicador específico de género: } 17.8 .1\end{array}$ \\
\hline
\end{tabular}

Como hemos indicado anteriormente, la perspectiva de género está presente en 11 de los ODS mediante la incorporación de los indicadores generados específicamente para cada uno de ellos, en mayor y menor medida. Sin embargo, existen 6 ODS que no introducen la perspectiva de género, aunque ésta sea imprescindible para el logro de las metas contenidas en dichos Objetivos. Éstos objetivos son: el relativo a Agua limpia y Saneamiento (6), el de Energía asequible y no contaminante (7), el de Industria, Innovación e Infraestructura (9), el de Producción y Consumo responsables (12), el de Vida marina (14) y el de Vida de ecosistemas terrestres (15).

Si bien podemos valorar positivamente la incorporación de las cuestiones relativas a la igualdad de género en los ODS con respecto a lo contenido en los ODM, todavía existen 6 ODS sin ningún indicador bajo la perspectiva de género. Con base en el análisis de los estudios e informes realizados hasta el momento sobre los avances de los ODS contenidos en la Agenda 2030 podemos identificar algunos de los retos que se están presentando en el desarrollo e implementación de las acciones concretas contenidas en cada uno de los 17 ODS. Entre las que podemos destacar las siguientes:

- La elaboración de indicadores con perspectiva de género en aquellos ODS que todavía no cuentan con ninguno y la ampliación de los mismos en aquellos en los que son insuficientes. Para mejorar la vida de millones de mujeres alrededor del mundo, se requiere de la elaboración de indicadores con perspectiva de género que permita evaluar la participación de la mujer en cada uno de los sectores de la sociedad. Promoviendo su empoderamiento y fomentando su labor en la imple- 
mentación de estrategias para la generación de desarrollo local e internacional. Ello permitirá también mejorar las condiciones de vida de millones de personas alrededor del mundo a la vez que permitirá promover los derechos humanos en su conjunto.

- La incorporación de lo transversal de la perspectiva de género en todas las políticas públicas diseñadas para el cumplimiento de los ODS. Para ello es necesario trabajar en la formación a los funcionarios públicos de las distintas áreas de gobierno para que puedan diseñar e implementar acciones concretas con enfoque de género, así como en la sociedad en general. Teniendo claro y bien definido que la base del desarrollo radica en la igualdad de hombres y mujeres. Siendo conscientes de que en la actualidad existen serias desigualdades sociales, económicas y políticas entre las mujeres y los hombres, ya que este reconocimiento permitirá identificar las principales problemáticas en cuestione de igualdad de género. Y que el empoderamiento de la mujer es en los diversos sectores de la sociedad es una pieza clave para el desarrollo. Reconociendo que las mujeres no son un grupo vulnerable como tal, sino que debido a las condiciones de exclusión y discriminación en las que se ven envueltas, pueden ser expuestas a una mayor vulnerabilidad. Y recordando siempre que para trabajar en la igualdad de género es imprescindible contar con la participación de los hombres, ya que son clave para construir una nueva cultura de igualdad, al introducir un cambio en las percepciones tradicionales sobre los roles de los hombres y las mujeres en la sociedad. Para finalmente poder desarrollar estrategias de desarrollo neutras en cuestiones de género en cada una de las esferas en las que se incorporen las metas contenidas en la Agenda 2030.

- La elaboración de indicadores específicos de género para los 17 ODS, mejorando la calidad, cobertura y disponibilidad de los datos en todos los países del mundo. La elaboración de los indicadores específicos de género deberá desarrollarse bajo en enfoque de los derechos humanos, que incluya las normativas y principios internacionales de los derechos humanos en los procesos de recopilación, análisis y divulgación de la información. Para ello se requiere de la participación de la población objetivo (la ciudadanía es una pieza clave en la generación eficaz de la información si se les incluye eficazmente), el desglose de la información con perspectiva de género, la autoidentificación de los datos y su punto de origen, la transparencia en la información, la confidencialidad y protección de los datos recopilados así como una buena rendición de cuentas, mediante la publicación imparcial de los resultados ${ }^{22}$.

${ }^{22}$ ONU MUJERES (2018) "Hacer las promesas realidad: la igualdad de género en la agenda 2030 para el desarrollo sostenible", p. 68. 


\section{Conclusiones}

La incorporación de los derechos de las mujeres en las normativas nacionales e internacional ha tenido grandes avances en las últimas décadas. Desde hace más de 60 años se han promulgado numerosas normas de alcance internacional para promover la participación de la mujer y la igualdad de género. Sin embargo, sigue existiendo una cultura soterrada de discriminación por cuestiones de género en la mayoría de los países del mundo. Como sabemos las mujeres menores de 40 años tienen una mayor probabilidad de sufrir algún tipo de pobreza que los hombres. Según las ultima cifras existen 4,4 millones de mujeres más que hombres que viven con menos de 1,90 dólares por día en 89 países del planeta, siendo la desigualdad de acceso a los recursos económicos una de las principales causas de esta problemática. El acceso de las mujeres al mercado laboral sigue siendo desigual y continúa estando fuertemente condicionado por las cuestiones culturales. De la misma forma se ve afectada la incorporación de las mujeres en la educación, existiendo un $48 \%$ de niñas que no asiste a la escuela en algunas regiones del mundo. La representación parlamentaria de las mujeres sigue siendo baja, pese a los recientes aumentos producidos en los primeros años de implementación de la Agenda 2030. Lo dificulta también la incorporación de estrategias claves para la igualdad de género si no se tiene esa visión femenina a la hora de elaborar las políticas nacionales.

En las cuestiones ambientales resulta de igual importancia la participación de las mujeres, ya que suelen ser las primeras afectadas por los desastres naturales y en sufrir las consecuencias económicas y sociales que eso trae consigo. Recordemos que son las mujeres y las niñas las que dedican muchas horas a la recolección de leña, forraje y agua para la limpieza de sus hogares y la preparación de alimentos. Asimismo, el tiempo dedicado al cuidado familiar les hace permanecer más tiempo en casa realizando trabajos no remunerados.

Es por ello que resulta de gran importancia incorporar el enfoque de género a cada uno de los 17 ODS contenidos en la Agenda 2030, ya que si logramos empoderar a las mujeres y niñas de todo el mundo y se alcanza una igualdad de género no solo estaremos contribuyendo a la construcción de un desarrollo humano sostenible, sino que estaremos promoviendo los derechos humanos en su conjunto. Ya que como sabemos la mujer es la pieza clave del desarrollo puesto que es ella la que fomenta en su seno familiar una serie de acciones y valores en cada uno de los miembros de su familia que a su vez interactuaran en la sociedad. Por lo que una mujer empoderada y con recursos económicos y materiales podrá mantener su hogar y su entorno en condiciones saludables (ODS1, ODS6, ODS7, ODS11, ODS12, ODS13, ODS14 y ODS15); podrá alimentar adecuadamente y proporcionar una educación de calidad a los miembros de su hogar (ODS2, ODS4); podrá cuidar su salud y la de los suyos y velar por la seguridad física, emocional y económica personal y familiar (ODS3, ODS16). Lo que se traducirá en la reducción de casos por mortalidad materno-infantil, en el incremento de la matriculación de la educación básica y superior; en el cuidado y manteniendo de los bosques, ríos y mares; así como en el incremento de la economía mundial por su valiosa participación en el mercado laboral (ODS5, ODS8, ODS9, ODS10). 
Mejorando con ello no solo sus condiciones de vida y la de los suyos sino su entorno y el de la sociedad en su conjunto. En definitiva, una mujer empoderada y valorada es el motor que aportará ese desarrollo humano sostenible que tanto se requiere.

\section{Bibliografía}

ASAMBLEA GENERAL DE NACIONES UNIDAS (2019) "Estudio mundial sobre el papel de la mujer en el desarrollo. Informe del secretario general. La importancia de hacer frente a la pobreza económica y la pobreza de tiempo de las mujeres en favor del desarrollo sostenible", 17 de junio de 2019, a/74/111, págs. 117.

ASAMBLEA GENERAL DE NACIONES UNIDAS (2018) “Las mujeres en el desarrollo”, Resolución 72/234, 20 de diciembre de 2017, a/res/72/234, págs. 17.

ASAMBLEA GENERAL DE NACIONES UNIDAS (2015) “Estudio mundial sobre el papel de la mujer en el desarrollo", Resolución 69/236, 19 de diciembre de 2014, a/res/69/236.

ASAMBLEA GENERAL DE NACIONES UNIDAS (2015) "Transformar nuestro mundo: la agenda 2030 para el desarrollo sostenible”, Resolución 70/1, 25 de septiembre de 2015, a/res/70/1.

BERNAL MARTÍNEZ DE SORIA, AURORA y CARRICA OCHOA, SARAH (2014) "Mujer, desarrollo y educación para el desarrollo", Revista Edetania: estudios y propuestas socioeducativas, issn 0214-8560, nº. 46, Págs. 181-200.

CALATRAVA, JAVIER (2002), “Mujer y desarrollo rural en la globalización: de los proyectos asistenciales a la planificación de género", Revista globalización y mundo rural, no. 803. P. 73.

FERNÁNDEZ SAAVEDRA ANA G. y DEMA MORENO SANDRA (2018) “La integración de la perspectiva de género en la gestión del riesgo de desastres: de los odm a los ods" Revista internacional de cooperación y desarrollo vol. 5 No. 1, Pág: 31-43.

FUNDACIÓN MUJERES (2003), “Guía para la elaboración de proyectos desde una perspectiva de género", Fondo Social Europeo y Gobierno del Principado de Asturias, p. 43.

HEIKEL, MARIA VICTORIAL (1994) "Mujer, población y desarrollo", población y desarrollo, issn-e 2076-054x, issn 2076-0531, no. 7, Págs. 31-37.

NACIONES UNIDAS (2019) “Informe de los objetivos de desarrollo sostenible, 2019”, Nueva York, 64.

NACIONES UNIDAS (1994) “Declaración y plataforma de acción de Beijing. Declaración política y documentos resultados de Beijing+5”, Nueva York, USA. Reimpreso en ONU Mujeres, Un women in 2014. Isbn: 978-1-936291-94-6. P. 316.

ONU MUJERES (2019) "Families in a changing world. Progress of the world's women 2019-2020.", Estados Unidos de América, págs. 285.

ONU MUJERES (2018) "Hacer las promesas realidad: la igualdad de género en la agenda 2030 para el desarrollo sostenible”, Estados Unidos de América, págs. 346.

ONU MUJERES (2018) “Informe anual 2017-2018 de onu mujeres”, Estados Unidos de América, págs. 52. 
RODRÍGUEZ MARTÍNEZ, ROCÍO (1994) “Mujer y desarrollo: reflexiones sobre el feminismo del norte y el desarrollo del sur: estudio especial sobre la mujer en América Latina”. América Latina hoy: Revista de Ciencias Sociales, issn 1130-2887, vol. 9, Págs. 31-36.

SCHOLTUS SILVIA C. y DOMATO 0. (2015) "El rol protagónico de la mujer en el desarrollo sustentable de la comunidad". Apuntes universitarios, issn-e 2225-7136, vol. 5, №. 1, (Ejemplar dedicado a: volumen v), págs. 9-34. 